\title{
OSCILLATION PROPERTIES OF PERTURBED DISCONJUGATE EQUATIONS
}

\author{
WILLIAM F. TRENCH
}

ABSTRACT. Oscillation conditions are given for the equation $L_{u}+f(t, u)=0$, where

$$
L u=\frac{1}{\beta_{n}} \frac{d}{d t} \frac{1}{\beta_{n-1}} \cdots \frac{d}{d t} \frac{1}{\beta_{1}} \frac{d}{d t} \frac{u}{\beta_{0}} \quad(n \geq 2),
$$

with $\beta_{0}, \ldots, \beta_{n}$ positive and continuous on $(0, \infty), \int^{\infty} \beta_{i} d t=\infty$ $(1 \leq i \leq n-1)$, and $f$ subject to conditions which include $u f(t, u)$ $\geq 0$. The results obtained include previously known oscillation conditions for the equation $u^{(n)}+f(t, u)=0$ for both linear and nonlinear cases.

We study the oscillation properties of the scalar equation

$$
L u+f(t, u)=0, \quad t>0,
$$

where

$$
L=\frac{1}{\beta_{n}} \frac{d}{d t} \frac{1}{\beta_{n-1}} \cdots \frac{d}{d t} \frac{1}{\beta_{1}} \frac{d}{d t} \frac{\dot{\beta}}{\beta_{0}} \quad(n \geq 2),
$$

with $\beta_{0}, \ldots, \beta_{n}$ continuous and positive on $(0, \infty)$. If $p_{1}, \ldots, p_{n}$ are continuous on $(0, \infty)$ and the equation

$$
L x \equiv x^{(n)}+p_{1}(t) x^{(n-1)}+\cdots+p_{n}(t) x=0
$$

is disconjugate on $(0, \infty)$, then $L$ can be rewritten as in (2), with $\beta_{i} \epsilon$ $C^{(n-i)}(0, \infty)[11]$. Thus, the class of operators (2) properly contains the normal disconjugate operators (3), as defined in [4].

Nehari [10] and Bogar [2] have studied the oscillation properties of (1) for the linear case, under assumptions that certain iterated integrals involving $\beta_{1}, \ldots, \beta_{n-1}$ diverge as $t \rightarrow \infty$. These assumptions are implied by the condition

$$
\int^{\infty} \beta_{i} d t=\infty, \quad 1 \leq i \leq n-1,
$$

which was assumed by $\mathrm{K}$ artsatos [5] in connection with a nonlinear case, and which, the author showed in [13], may be imposed without loss of generality; i.e., (2) can always be rewritten so that (4) holds, and this requirement determines $\beta_{0}, \ldots, \beta_{n}$ uniquely up to positive multiplicative constants

Received by the editors March 22, 1974 and, in revised form, June 25, 1974. AMS (MOS) subject classifications (1970). Primary 34C10, 34C11.

Key words and phrases. Oscillation, disconjugate. 
with product one. Henceforth we assume (4).

If $\alpha_{1}, \alpha_{2}, \ldots$ are continuous on $(0, \infty)$ and $c>0$, define $I_{0}=1$ and

$$
I_{r}\left(t, c ; a_{r}, \ldots, \alpha_{1}\right)=\int_{c}^{t} a_{r}(\lambda) I_{r-1}\left(\lambda, c ; \alpha_{r-1}, \ldots, \alpha_{1}\right) d \lambda, \quad r \geq 1 .
$$

Repeated integration by parts yields

$$
I_{r}\left(t, c ; a_{r}, \ldots, a_{1}\right)=(-1)^{r} I_{r}\left(c, t ; a_{1}, \ldots, a_{r}\right)
$$

[14, Lemma 2.2], which we will use later.

The functions

$$
\tilde{x}_{j}(t)=\beta_{0}(t) I_{j-1}\left(t, c ; \beta_{1}, \ldots, \beta_{j-1}\right), \quad 1 \leq j \leq n,
$$

form a fundamental set for $L_{x}=0$ such that $\tilde{x}_{j}(t)>0$ for large $t$, and, because of $(4), \lim _{t \rightarrow \infty} \tilde{x}_{i}(t) / \tilde{x}_{j}(t)=0,1 \leq i<j \leq n$. Such a fundamental set is called a principal system for $L x=0$. It has been shown by Hartman [4], Levin [7] and Willett [14] that a normal disconjugate equation (3) has principal systems, and the author showed [13] (without assuming (4) at the outset) that this is also true of $L x=0$ with $L$ as in (2).

The adjoint of (2) is

$$
L^{*}=\frac{1}{\beta_{0}} \frac{d}{d t} \frac{1}{\beta_{1}} \cdots \frac{d}{d t}-\frac{1}{\beta_{n-1}} \frac{d}{d t} \frac{\dot{ }}{\beta_{n}}
$$

(Nehari [10]), and the functions

$$
\tilde{y}_{j}(t)=\beta_{n}(t) i_{j-1}\left(t, c ; \beta_{n-1}, \ldots, \beta_{n-j+1}\right), \quad 1 \leq j \leq n,
$$

form a principal system for $L^{*} y=0$.

We define the operators $L_{0}, \ldots, L_{n}$ by $L_{0} x=x / \beta_{0}$ and

$$
L_{j} x=\frac{1}{\beta_{j}} \frac{d}{d t} \frac{1}{\beta_{j-1}} \cdots \frac{d}{d t}-\frac{1}{\beta_{1}} \frac{d}{d t} \frac{x}{\beta_{0}}, \quad 1 \leq j \leq n,
$$

so that

$$
\left(L_{j-1} x\right)^{\prime}=\beta_{j} L_{j} x, \quad 1 \leq j \leq n
$$

and $L_{n}=L$. An extendible solution of (1) is a function $u$ such that $L_{0} u, \ldots$, $L_{n} u$ exist and satisfy $(1)$ on some interval $\left(t_{0}, \infty\right)$. An extendible solution is oscillatory if it has infinitely many zeros on every such interval.

Lemma 1. Suppose $u(t)>0$ and $L_{n} u(t) \leq 0$ for $t \geq t_{0}$. Then

(a) if $n$ is even, there is an even integer $k$ such that $0 \leq k \leq n-2$,

$$
\begin{gathered}
L_{j} u(t)>0 \quad \text { for large } t, \quad 0 \leq j \leq k, \\
\left.\lim _{t \rightarrow \infty} L_{k+1} u(t)=\alpha \quad \text { (finite, } \geq 0\right),
\end{gathered}
$$


and

$$
\lim _{t \rightarrow \infty} L_{j} u(t)=0, \quad k+2 \leq j \leq n-1
$$

(b) if $n$ is odd, either

$$
\lim _{t \rightarrow \infty} L_{j} u(t)=0, \quad 1 \leq j \leq n-1
$$

or there is an odd integer $k$ such that (8), (9) and (10) hold.

This lemma is an adaptation of a result of Kneser [6]. Its proof is by separate inductions for even and odd $n$, and leans heavily on the fact that if $(-1)^{s} \lim _{t \rightarrow \infty} L{ }_{j} u(t) \geq \epsilon>0$ for some $j \geq 1$, then (4) and repeated integration of (7) yield $(-1)^{s} \lim _{t \rightarrow \infty} L_{0} u(t)=\infty$.

Let $\left\{x_{1}, \ldots, x_{n}\right\}$ and $\left\{y_{1}, \ldots, y_{n}\right\}$ be principal systems for $L_{x}=0$ and $L^{*} y=0$, and let $z_{i}$ be a solution of $L_{i}\left(x_{1} z_{i}\right)=y_{n-i} / y_{1}, 0 \leq i \leq n-2$. It can be shown that if $t_{1}>0$, then

$$
x_{i}(t)=\beta_{0}(t) \sum_{j=1}^{i} a_{i j}{ }_{j-1}\left(t, t_{1} ; \beta_{1}, \ldots, \beta_{j-1}\right)
$$

and

$$
y_{i}(t)=\beta_{n}(t) \sum_{j=1}^{i} b_{i j}{ }_{j-1}\left(t, t_{1} ; \beta_{n-1}, \ldots, \beta_{n-j+1}\right) \text {, }
$$

where $a_{i j}$ and $b_{i j}$ are constants, $a_{i i}>0$ and $b_{i i}>0$. From (4), (12), (13) and L'Hospital's rule,

and

$$
\lim _{t \rightarrow \infty} \frac{\beta_{0}(t) I_{i-1}\left(t, t_{1} ; \beta_{1}, \ldots, \beta_{i-1}\right)}{x_{i}(t)}=a_{i i}^{-1}>0
$$

$$
\lim _{t \rightarrow \infty} \frac{\beta_{n}(t) I_{i-1}\left(t, t_{1} ; \beta_{n-1}, \ldots, \beta_{n-i+1}\right)}{y_{i}(t)}=b_{i i}^{-1}>0
$$

moreover, it can be shown that

$$
\lim _{t \rightarrow \infty} \frac{I_{n-1}\left(t, t_{1} ; \beta_{n-1}, \ldots, \beta_{1}\right)}{z_{0}(t)}=c_{0}>0 \quad \text { (finite) }
$$

and

$$
\begin{aligned}
& \lim _{t \rightarrow \infty} \frac{I_{n-1}\left(t, t_{1} ; \beta_{1}, \ldots, \beta_{k}, \beta_{n-1}, \ldots, \beta_{k+1}\right)}{z_{k}(t)}=c_{k}>0 \quad \text { (finite), } \\
& 1 \leq k \leq n-2 .
\end{aligned}
$$

The last four equations imply that if the assumptions of Theorems 1 and 2 hold for a given choice of principal systems for $L x=0$ and $L^{*} y=0$, then they hold for all choices. 
Theorem 1. Suppose $f$ is continuous on $(0, \infty) \times(-\infty, \infty)$,

$$
u f(t, u) \geq 0
$$

and

$$
|f(t, u)| \geq h(t) \phi\left(|u| / \beta_{0}(t)\right)
$$

where $h$ is continuous and nonnegative and $\phi$ is nondecreasing and positive on $(0, \infty)$. Suppose also that

$$
\int_{n-k-1}^{\infty}(s) h(s) \phi\left(\frac{\rho x_{k+1}(s)}{x_{1}(s)}\right) d s=\infty
$$

for every positive $\rho$ and nonnegative integer $k$ such that $n-k$ is even and positive. Then (a) if $n$ is even, every extendible solution of (1) is oscillatory; (b) if $n$ is odd and $u$ is a nonoscillatory extendible solution of (1), then $\lim _{t \rightarrow \infty} L_{j} u(t)=0(1 \leq j \leq n-1)$ and $\lim _{t \rightarrow \infty} L_{0} u(t)=\gamma$ (finite). In this case, $\gamma=0$ if

$$
\int^{\infty} y_{n}(s) h(s) d s=\infty .
$$

Proof. Suppose $u$ is an extendible solution of (1) which is positive on $\left(t_{0}, \infty\right)$ and satisfies (8), (9) and (10) for the integer $k$ indicated in Lemma

1. Then (7), (9) and (10) yield

$$
L_{k+1} u(t)=\alpha+\int_{t}^{\infty} I_{n-k-2}\left(t, s ; \beta_{k+2}, \ldots, \beta_{n-1}\right) \beta_{n}(s) f(s, u(s)) d s,
$$

and, since $n-k-2$ is even, (18) implies that the integrand is nonnegative; therefore,

$$
L_{k+1} u(t) \geq \alpha, \quad t \geq t_{0} .
$$

The absolute convergence of the integral in (22), together with (6), (15) and (19), implies that

$$
\int_{n-k-1}^{\infty} y(s) h(s) \phi\left(L_{0} u(s)\right) d s<\infty .
$$

But (7) and (23) imply that $\left(L_{k} u\right)^{\prime} \geq 0$; hence, from (8), $L_{k} u(t) \geq R$ for some positive $R$ and $t$ sufficiently large, say $t \geq t_{1}$. Repeated integration of (7) for $j=k, \ldots, 1$ yields

$$
\begin{aligned}
L_{0} u(t) \geq & R I_{k}\left(t, t_{1} ; \beta_{1}, \ldots, \beta_{k}\right) \\
& +\sum_{j=0}^{k-1} L_{j} u\left(t_{1}\right) I_{j}\left(t, t_{1} ; \beta_{1}, \ldots, \beta_{j}\right), \quad t \geq t_{1} .
\end{aligned}
$$


From (4) and L'Hospital's rule,

$$
\lim _{t \rightarrow \infty} \frac{I_{j}\left(t, t_{1} ; \beta_{1}, \ldots, \beta_{j}\right)}{I_{k}\left(t, t_{1} ; \beta_{1}, \ldots, \beta_{k}\right)}=0, \quad 0 \leq j<k
$$

hence, (25) implies that

$$
L_{0} u(t) \geq(R / 2) I_{k}\left(t, t_{1} ; \beta_{1}, \ldots, \beta_{k}\right) \text { for large } t,
$$

and therefore, from (14),

$$
\liminf _{t \rightarrow \infty} \frac{L_{0} u(t)}{x_{k+1}(t) / x_{1}(t)}>0 .
$$

This and (24) imply that the integral in (20) converges for some positive $\rho$, a contradiction.

It now follows from Lemma 1 that (1) has no extendible positive solutions except, possibly, some which satisfy (11), if $n$ is odd. For such a solution, $(-1)^{n-j} L_{j} u(t) \leq 0(1 \leq j \leq n)$ for large $t$, so that $L_{1} u(t) \leq 0$ and $L_{0} u(t)$ is decreasing and positive for large $t$; hence,

$$
L_{0} u(t) \geq \gamma=\lim _{t \rightarrow \infty} L_{0} u(t) \geq 0 .
$$

From (7), (11) and (26),

$$
L_{0} u(t)=\gamma+\int_{t}^{\infty} I_{n-1}\left(t, s ; \beta_{1}, \ldots, \beta_{n-1}\right) \beta_{n}(s) f(s, u(s)) d s .
$$

If $\gamma>0$, then $f(s, u(s)) \geq h(s) \phi(\gamma)$, and the convergence of the integral in (27) implies that

$$
\int_{t}^{\infty} I_{n-1}\left(t, s ; \beta_{1}, \ldots, \beta_{n-1}\right) \beta_{n}(s) b(s) d s<\infty,
$$

which, because of (6) and (15), implies that the integral in (21) converges.

This completes the proof of Theorem 1 as far as positive solutions are concerned. Since $f$ satisfies (18) and Lemma 1 also holds with all of its inequalities reversed, the discussion of negative solutions proceeds in the same way.

Theorem 1 contains the following known special case.

Corollary 1 (Anan' eva and Balaganskii [1]). If $p$ is positive and continuous on $(0, \infty)$ and

$$
\int^{\infty} s^{n-2} p(s) d s=\infty
$$

then every solution of $y^{(n)}+p(t) y=0, t>0$, is oscillatory if $n$ is even and, if $n$ is odd, every nonoscillatory solution must approach zero monotonically as $t \rightarrow \infty$, along with its first -1 derivatives. 
(Anan'eva and Balaganskii also considered in [1] the oscillation properties of (1), subject to (2), (4) and (18), but with other conditions on $f$ different from ours, and obtained a result which contains Fite's oscillation theorem [3, Theorem V], but not Corollary 1.)

The next theorem is related to results of Ryder and Wend [12] for the special case where $L u=u^{(n)}$, under assumptions which rule out linearity for (1).

Theorem 2. Suppose $f$ satisfies the assumptions of Theorem 1, except for (20). Then the conclusions of Theorem 1 hold if (a)

$$
\int_{\rho}^{\infty}(\phi(r))^{-1} d r<\infty, \quad 0<\rho<\infty
$$

and

$$
\int^{\infty} y_{1}(s) z_{k}(s) h(s) d s=\infty
$$

for all integers $k$ such that $n-k$ is even and positive; or if (b)

$$
\begin{array}{cc}
\phi\left(r_{1} r_{2}\right) \geq \phi\left(r_{1}\right) \phi\left(r_{2}\right), & r_{1}, r_{2}>0, \\
\int_{0}^{\rho}(\phi(r))^{-1} d r<\infty, & 0<\rho<\infty,
\end{array}
$$

and

$$
\int^{\infty} y_{1}(s) h(s) \phi\left(z_{k}(s)\right) d s=\infty
$$

for all such $k$.

Proof. As in the proof of Theorem 1, it suffices to consider positive solutions. Suppose $u$ is a positive solution of $(1)$ on $\left(t_{0}, \infty\right)$ which satisfies (8), (9) and (10) for the integer $k$ indicated in Lemma 1 . Since $\alpha \geq 0$, (19) and (22) imply that

$$
L_{k+1} u(t) \geq \int_{t}^{\infty} I_{n-k-2}\left(t, s ; \beta_{k+2}, \ldots, \beta_{n-1}\right) V(s) d s, \quad t \geq t_{0},
$$

where

$$
V(s)=\beta_{n}(s) b(s) \phi\left(L_{0} u(s)\right)
$$

Now suppose (8) is satisfied for $t \geq t_{1}$. Multiplying (34) by $\beta_{k+1}$, integrating from $t_{1}$ to $t$, and changing the order of integration yields

$$
\begin{aligned}
L_{k} u(t)> & \int_{t_{1}}^{t} V(s) d s \int_{t_{1}}^{s} \beta_{k+1}(\lambda) I_{n-k-2}\left(\lambda, s ; \beta_{k+2}, \ldots, \beta_{n-1}\right) d \lambda \\
& +\int_{t}^{\infty} V(s) d s \int_{t_{1}}^{t} \beta_{k+1}(\lambda) I_{n-k-2}\left(\lambda, s ; \beta_{k+2}, \ldots, \beta_{n-1}\right) d \lambda, \quad t \geq t_{1} .
\end{aligned}
$$


Since $n-k-2$ is even,

$I_{n-k-2}\left(\lambda, s ; \beta_{k+2}, \ldots, \beta_{n-1}\right) \geq I_{n-k-2}\left(\lambda, t ; \beta_{k+2}, \ldots, \beta_{n-1}\right), \quad \lambda \leq t \leq s$.

Substituting the right side in the second integral in (36) and using (5) and (6) yields

$$
\begin{aligned}
L_{k} u(t)> & \int_{t_{1}}^{t} I_{n-k-1}\left(s, t_{1} ; \beta_{n-1}, \ldots, \beta_{k+1}\right) V(s) d s \\
& +I_{n-k-1}\left(t, t_{1} ; \beta_{n-1}, \ldots, \beta_{k+1}\right) \int_{t}^{\infty} V(s) d s, \quad t \geq t_{1} .
\end{aligned}
$$

If $k=0$, this gives a lower bound for $L_{0} u(t)$; if $k \geq 1$, we ignore the first integral in (37). Then (7), (8) and (37) yield

$$
L_{k-1} u(t)>\int_{t_{1}}^{t} \beta_{k}(\lambda) I_{n-k-1}\left(\lambda, t_{1} ; \beta_{n-1}, \ldots, \beta_{k+1}\right) d \lambda \int_{\lambda}^{\infty} V(s) d s .
$$

Changing the order of integration and using (5) yields

$$
L_{k-1} u(t)>\int_{t_{1}}^{t} I_{n-k}\left(s, t_{1} ; \beta_{k}, \beta_{n-1}, \ldots, \beta_{k+1}\right) V(s) d s
$$

$$
+I_{n-k}\left(t, t_{1} ; \beta_{k}, \beta_{n-1}, \ldots, \beta_{k+1}\right) \int_{t}^{\infty} V(s) d s, \quad t \geq t_{1} .
$$

If $k=1,(38)$ gives a lower bound for $L_{0} u(t)$; if $k>1$, repetition of the argument that led from (37) to (38) yields finally the inequality

$$
\begin{aligned}
L_{0} u(t)> & \int_{t_{1}}^{t} w_{k}(s) \beta_{n}(s) h(s) \phi\left(L_{0} u(s)\right) d s \\
& +w_{k}(t) \int_{t}^{\infty} \beta_{n}(s) b(s) \phi\left(L_{0} u(s)\right) d s, \quad t \geq t_{1},
\end{aligned}
$$

after recalling (35) and defining

$$
w_{k}(t)= \begin{cases}I_{n-1}\left(t, t_{1} ; \beta_{n-1}, \ldots, \beta_{1}\right), & k=0, \\ I_{n-1}\left(t, t_{1} ; \beta_{1}, \ldots, \beta_{k}, \beta_{n-1}, \ldots, \beta_{k+1}\right), & 1 \leq k \leq n-2 .\end{cases}
$$

If (a) of Theorem 2 holds, call the first integral in (39) $F(t)$ and ignore the second. We employ a device used by Macki and Wong [9] and Ryder and Wend [12]: since $\phi$ is nondecreasing and $F(t)>0$ for large $t$ (say $t \geq t_{2}$ ), (39) implies that $\phi\left(L_{0} u(t)\right) / \phi(F(t)) \geq 1, t \geq t_{2}$. (Since $h(t) \geq 0$ by assumption, $F(t) \equiv 0$ would imply that $h(t) \equiv 0$, contradicting (30).) Therefore,

$$
F^{\prime}(t) / \phi(F(t)) \geq \beta_{n}(t) w_{k}(t) h(t), \quad t \geq t_{2},
$$

and

$$
\int_{F\left(t_{2}\right)}^{F(t)}(\phi(r))^{-1} d r \geq \int_{t_{2}}^{t} \beta_{n}(s) w_{k}(s) h(s) d s, \quad t \geq t_{2}
$$


Hence, (29) implies that the integral on the right converges as $t \rightarrow \infty$. However, from (13), (16), (17) and (40),

$$
\lim _{s \rightarrow \infty} w_{k}(s) / z_{k}(s)=c_{k} \text { and } y_{1}(s)=b_{11} \beta_{n}(s),
$$

and therefore the integral (30) is convergent, a contradiction. Hence, the conclusions of Theorem 1 hold under the assumptions of Theorem 2(a).

If (b) of Theorem 2 holds, call the second integral in (39) $H(t)$ and ignore the first; then, from (31),

$$
\phi\left(L_{0} u(t)\right) / \phi(H(t)) \geq \phi\left(w_{k}(t)\right), \quad t \geq t_{1},
$$

and therefore

$$
-H^{\prime}(t) / \phi(H(t)) \geq \beta_{n}(t) h(t) \phi\left(w_{k}(t)\right)
$$

and

$$
\int_{H(t)}^{H\left(t_{1}\right)}(\phi(r))^{-1} d r \geq \int_{t_{1}}^{t} \beta_{n}(s) h(s) \phi\left(w_{k}(s)\right) d s .
$$

Now (32) implies that the integral on the right converges as $t \rightarrow \infty$, which, because of (31) and (41), contradicts (33). Thus, the conclusions of Theorem 1 hold under the as sumptions of Theorem 2(b).

For the special case where $L x=x^{(n)},(30)$ is equivalent to $\int^{\infty} s^{n-1} h(s) d s=\infty$, and Theorem 2(a) is related to Theorem 1 of Ryder and Wend [12], and is a generalization of the sufficiency half of Theorem 2 of Ličko and Švec [8] for the equation

$$
y^{(n)}+h(t)|y|^{\gamma} \operatorname{sgn} y=0 \quad(h>0),
$$

with $\gamma>1$. The proof of Theorem 2(a) is based on the proof of Ryder and Wend. Because of (31), (33) is equivalent to $\int^{\infty} h(s) \phi\left(s^{n-1}\right) d s=\infty$ if $L x$ $=x^{(n)}$, so that Theorem 2(b) is related to Theorem 2 of Ryder and Wend (but the proofs are different), and is a generalization of the sufficiency half of Theorem 1 of Ličko and Švec [8] for (42), with $0<y<1$.

\section{REFERENCES}

1. G. B. Anan'eva and V. I. Balaganskii, Oscillation of the solutions of certain differential equations of high order, Uspehi Mat. Nauk 14 (1959), no. 1 (85), 135-140. (Russian) MR 21 \#1428.

2. G. A. Bogar, Oscillation properties of two term linear differential equations, Trans. Amer. Math. Soc. 161 (1971), 25-33. MR 44 \#1870.

3. W. B. Fite, Concerning the zeros of the solutions of certain differential equations, Trans. Amer. Math. Soc. 19 (1918), 341-352.

4. P. Hartman, Principal solutions of disconjugate $n$-th order linear differential equations, Amer. J. Math. 91 (1969), 306-362. MR 40 \#450.

5. A. G. Kartsatos, Oscillation properties of even order differential equations, Bull. Fac. Sci. Ibaraki Univ. Ser. A No. 2-1 (1969), 9-14. MR $40 \# 7536$. 
6. A. Kneser, Untersuchungen über die reelen Nullstellen der Integrale linearer Differentialgleichen, Math. Ann. 42 (1893), 409-435.

$7 . A_{1}$ Ju. Levin, The non-oscillation of solutions of the equation $x^{(n)}+$ $p_{1}(t) x^{(n-1)}+\ldots+p_{n}(t) x=0$, Uspehi Mat. Nauk $24(1969)$, no. $2(146), 43-96=$ Russian Math. Surveys 24 (1969), no. 2, 43-96. MR 40 \#7537.

8. I. Ličko and M. Švec, Le caractère oscillatoire des solutions de l'équation $y^{(n)}+f(x) y^{a}=0, n>1$, Czechoslovak Math. J. 13 (88) (1963), 481-491. MR 28 \#4210.

9. J. W. Macki and J. S. W. Wong, Oscillation of solutions to second-order nonlinear differential equations, Pacific J. Math. 24 (1968), 111-117. MR 37 \#507.

10. Z. Nehari, Non-oscillation criteria for $n$-th order linear differential equations, Duke Math. J. 32 (1965), 607-615. MR $32 \# 4338$.

11. G. Polya, On the mean-value theorem corresponding to a given linear homogeneous differential equation, Trans. Amer. Math. Soc. 24 (1924), 312-324.

12. G. H. Ryder and D. V. V. Wend, Oscillation of solutions of certain ordinary differential equations of nth order, Proc. Amer. Math. Soc. 25 (1970), 463-469.

MR 41 \#5710.

13. W. F. Trench, Canonical forms and principal systems for general disconjugate equations, Trans. Amer. Maţh. Soc. 189 (1974), 319-327.

14. D. Willett, Asymptotic behaviour of disconjugate nth order differential equations, Canad. J. Math. 23 (1971), 293-314.

DEPARTMENT OF MATHEMATICS, DREXEL UNIVERSITY, PHIL ADELPHIA, PENNSYLVANIA 19104 